\title{
vTaiwan: An Empirical Study of Open Consultation Process in Taiwan
}

\author{
Yu-Tang Hsiao \\ Public Digital Innovation Space \\ Taipei, Taiwan \\ olivebranch.a@gmail.com
}

\author{
Short Paper \\ Shu-Yang Lin \\ Public Digital Innovation Space \\ Taipei, Taiwan \\ shuyanglin@ey.gov.tw
}

\author{
Audrey Tang \\ Public Digital Innovation Space \\ Taipei, Taiwan \\ audreyt@audreyt.org
}

\author{
Darshana Narayanan \\ Polis \\ New York, United States \\ darshana@dznarayanan.com
}

\author{
Claudina Sarahe \\ Composites Collective \\ New York, United States \\ cs@compositescollective.com
}

\begin{abstract}
Launched in 2014, vTaiwan is an open consultation process that brings Taiwan citizens and government together in online and of fline spaces, to deliberate and reach rough consensus on national issues, and to craft national digital legislation. This paper documents vTaiwan's background, open consultation process framework, and the collaborative open source engagement tools used in vTaiwan. The UberX case study highlights vTaiwan's open format and crowdsourcing characteristics as well as vTaiwan's culture-a recursive public that shapes an interactive environment that is capable of speaking to existing forms of power through an evolving rough consensus. Aiming to go beyond its limitations and challenges, vTaiwan iterates on creating a feasible model of decentralized consultation for society[17].
\end{abstract}

\section{CCS CONCEPTS}

•Human-centered computing $\rightarrow$ Participatory design; Empirical studies in interaction design;

\section{KEYWORDS}

vTaiwan, rulemaking, open consultation process

\section{ACM Reference format:}

Yu-Tang Hsiao, Shu-Yang Lin, Audrey Tang, Darshana Narayanan, and Claudina Sarahe. 2018. vTaiwan: An Empirical Study of Open Consultation Process in Taiwan. In Proceedings of OOO, OOO, OOO, 5 pages.

DOI: $10.475 / 123 \_4$

\section{INTRODUCTION}

In Taiwan, the 1990s were characterized by two synchronized developments: the democratic reforms, which followed the end of the martial law in 1987 and the information revolution, spurred

Permission to make digital or hard copies of part or all of this work for personal or classroom use is granted without fee provided that copies are not made or distributed for profit or commercial advantage and that copies bear this notice and the full citation on the first page. Copyrights for third-party components of this work must be honored. For all other uses, contact the owner/author(s).

OOO, OOO

(c) 2018 Copyright held by the owner/author(s). $123-4567-24-567 / 08 / 06 \ldots \$ 15.00$ DOI: $10.475 / 123 \_4$ by the advent of new information and communication technologies, both of which served to significantly transform the Taiwanese social and political landscape. In this context, debates on citizen oversight and participation in Taiwan have since the 1990s been closely linked to discussions on e-government and disclosure of government information[37]. Citizen participation in democratic Taiwan developed in several formats, both at the national and local levels: from institutionalized citizen participation-e.g. election procedures, public hearings, and more recently, government technologies (gov tech) that promote citizen participation; to noninstitutionalized participation-e.g. citizen-led social movements, open source civic technologies (civic tech).

During the Ma Ying-jeou administration (2008-2016), a number of social movements succeeded one another and served as the main social resilience practice to contest government actions[23]. Despite the growing demand for citizen participation, Taiwanese political sphere still lacked an institutionalized policy-making deliberative space and an open consultation process where citizens could engage in dialogue with the public and private sectors to reach rough consensus on issues in the public sphere and help government craft public policies. The climate of discontent and need for a practice of open consultation reached its paroxysm with the Sunflower Movement in 2014. From March $18^{\text {th }}$ to April $10^{\text {th }}$, citizens occupied the Legislative Yuan (Parliament) in Taipei to protest the opacity of the Cross-Strait Service Trade Agreement that aimed at liberalizing trade in services between Taiwan and China. During this occupation, Sunflower activists engaged Taiwan civil society in large-scale public deliberations and consultations. As a consequence, the ruling party Kuomintang (KMT) lost all its authority, as well as its legitimacy to act. Furthermore, the Sunflower Movement led to a revival of citizen participation. In the time following, government and civil society organizations in Taiwan have developed many projects to include citizens in decision-making processes. For example-the vTaiwan project in 2014[16], Join platform in 2015[7] and Participatory Budgeting in 2016[35].

This paper concentrates on vTaiwan, an open consultation process that brings Taiwan citizens and government together to craft country-wide digital legislation. 


\section{VTAIWAN BACKGROUND}

In December 2014, the then Minister without Portfolio of the Executive Yuan, Jaclyn Tsai, attended a hackathon organized by g0v[5]the largest civic tech community in Asia-and requested g0v contributors to build a platform that would allow the entire Taiwan society to engage in "rational discussion" [14] of public matters at a national scale. Tsai's proposal kick-started vTaiwan. The " $v$ " in vTaiwan stands for "vision", "voice", "vote" and "virtual", as vTaiwan embarks on a "virtual venture" of open consultation processes with the citizens.

vTaiwan aims to go beyond political polarization[28], to break out of echo chambers generated by social media[20] [36] and to reach rough consensus among stakeholders. In contrast to "democracy of rejection" in which social movements shaped by "negative coalitions [or] reactive majorities [...] [who] can tolerate their own contradictions more easily", vTaiwan participates in the construction of a "democracy of proposition" where the social majority "cannot be based on equivocation or ambiguity [but] presuppose[s] a positive and deliberate agreement."[32]

Participation in rulemaking has been a vibrant research topic for the past decades. Participatory rulemaking aims to facilitate the inclusion of individuals or groups in the design of policies[31] Riding on the advancement of technology, digital transformation in governments has not only digitized documents and papers but also opened up the government, achieved new levels of transparency, and allowed people inside and outside government to innovate on a shared platform. The vTaiwan process was developed by drawing on world partners' efforts in experimenting with new technology and the internet to facilitate public participation.[19] One of these projects is the Regulation Room (RegR)[33], operated by the Cornell E-Rulemaking Initiative[1]. RegR provides an online environment to facilitate public discussion and feedback on proposed federal rules. vTaiwan drew from the RegR's goal to broaden participation and improve quality in rulemaking using technology. vTaiwan built on the valuable work of RegR and further designed itself as a recursive public[27] that is open to transformation and reformulation. vTaiwan therefore empowers the public to define the topic of discussion, the agenda of how a topic is being discussed, and the tools used to facilitate the conversation. vTaiwan is run by the Coherent Blended Volition (CBV)[24] of participants and contributors; this approach emphasizes a "conceptual blend" of diverse views by incorporating the most essential elements of the different perspectives into a coherent whole.

\section{VTAIWAN PROCESS}

The vTaiwan process consists of four successive stages: proposal, opinion, reflection and legislation. There is no strict policy in the vTaiwan process to move from one stage to the next. The transitions between stages are decided by consensus from the vTaiwan community. This open format principle enables meaningful deliberation when all stakeholders are ready and willing to collaborate and iterate on solutions. The methodology of the participant-oriented agenda and rolling correction substantially engages citizens and public servants.

Every vTaiwan issue has a facilitator who guides the issue through the entire process. The facilitator uses the Focused Conversation

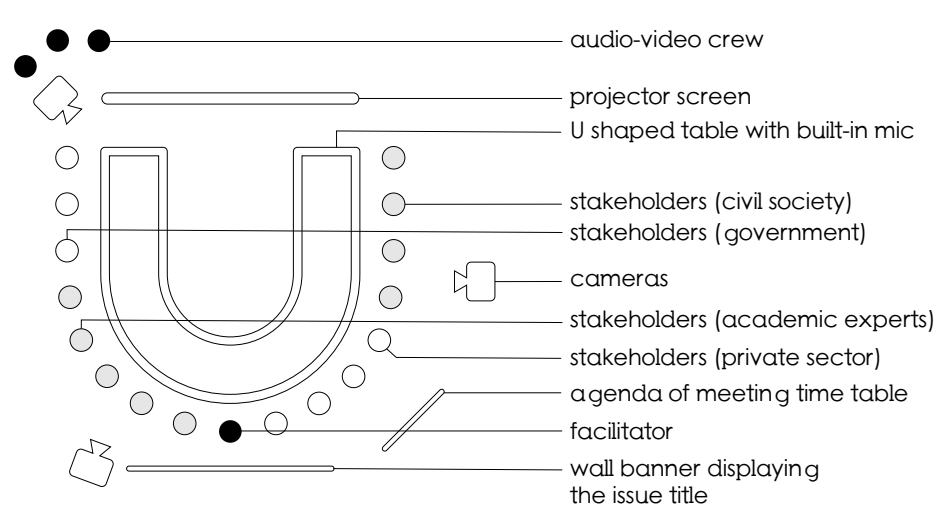

Figure 1: Layout of the meeting room.

Method (ORID)[34] to lead discussions at all stages of the process, both online and offline. The ORID method helps a facilitator not only to carefully analyse people's comments but also to scrutinize their own perceptions. O stands for the "objective" level, where participants share facts and data; R represents the "reflective" level, in which participants express their emotions or feelings about the objective facts; I means the "interpretive" level, where participants exchange opinions and values; and D signifies the "decisional" level, in which participants reach decisions and consensuses.

\subsection{Stage 1: Proposal Stage}

vTaiwan hosts weekly mini hackathons-an online-offline open community taking shape as a hackathon-to welcome all opinions from all walks of life, including programmers, designers, public servants, journalists, scholars, legal specialists, students and so on. At the mini hackathon, contributors submit an issue to a competent government authority who has the choice to accept (be accountable for the issue) or refuse to open the proposal topic. An issue will not move into the vTaiwan process without a government authority being accountable for the issue and a facilitator taking charge of the issue. In this stage, open source collaborative real-time text editors, such as Hackpad[6], serve the purpose of sharing notes taken at mini hackathons, meanwhile tools for shared documents and media storage, such as SlideShare[12], are used by all stakeholders to inform themselves about the issue under discussion.

\subsection{Stage 2: Opinion Stage}

After ensuring accountability, vTaiwan initiates the opinion stage to launch online opinion collection. vTaiwan first conducts an online survey with stakeholders within the community's network, who could potentially recommend other stakeholders in their network. Then online opinion is collected using Discourse[4], Pol.is[9], Typeform[15] and Sli.do[13]. Discourse, an open source discussion platform, enhances accountability by requiring competent authorities to reply to stakeholders' comments within seven days. Pol.is facilitates open-ended engagement from large groups of people and clusters participants into opinion groups to illuminate the critical aspects of the topic in real-time, using Principal Components 
Analysis[26][18] and K-means clustering[29]. Online opinion collection may take one or several rounds of opinion surveys. Each round of opinion collection lasts for at least one month, but vTaiwan does not set a limit to the number of rounds.

\subsection{Stage 3: Reflection Stage}

In this stage, the facilitator hosts an online-offline in-person consultation with stakeholders, including scholars, public servants, private sector representatives and community participants. The layout of the physical meeting space is designed to promote participation and deliberation (Figure 1). The consultation is livestreamed and the facilitator simultaneously prompts the in-person discussion and relays the online discussion to the physical space. LIVEhouse.in[8] and YouTube are the main platforms used to broadcast the livestreamed consultation. LIVEhouse.in also has a chat room for remote participants.

The meeting starts with the facilitator giving a summary of the process so far. Presentations from the stakeholder groups follow. The facilitator uses digital note-taking to summarize information in real-time and document. All information is displayed on the projector screen. The online discussion administrators actively monitor the feed for questions and comments on LIVEhouse.in Videos are released on the vTaiwan Facebook page where more citizens continue to engage throughout the following weeks. The digital tools provide all citizens with channels to have a voice and participate online, overcoming the obstacles of time and space, enabling civic engagement, and allowing divergence and convergence of idea flows until consensus is reached.

\subsection{Stage 4: Legislation Stage}

vTaiwan then moves to the legislation stage and presents the consensus on the policy or legislative solutions. In some cases, the issue is resolved with a guideline, a policy or a statement by the competent authority. In other cases, it could be formulated into a draft bill sent to the Legislative Yuan.

The vTaiwan process has three key mechanisms for public participation -interpretation, facilitation and documentation-supported by a selection of collaborative open source engagement tools (Figure 2). Interpretation mechanism emphasizes the importance to bridge communication gaps among people from diverse backgrounds by interpreting terminologies, jargons and vocabularies in various contexts. Facilitation mechanism draws the overall engagement from conflicts to consensus. Interpretation is for veterans to get the ideas across to the laymen, while facilitation is the art of collecting opinions from the public. Documentation mechanism highlights the importance of transparency and accountability. Transparency-of the process as well as the data-allows participants to understand the decisions taken through the vTaiwan process. Transparency also contributes to the establishment and elaboration of trust in the vTaiwan process and strengthens the legitimacy of the decisions taken throughout the four stages of vTaiwan.

To date, 26 national issues have been discussed through the vTaiwan open consultation process, and more than $80 \%$ have led to decisive government action.

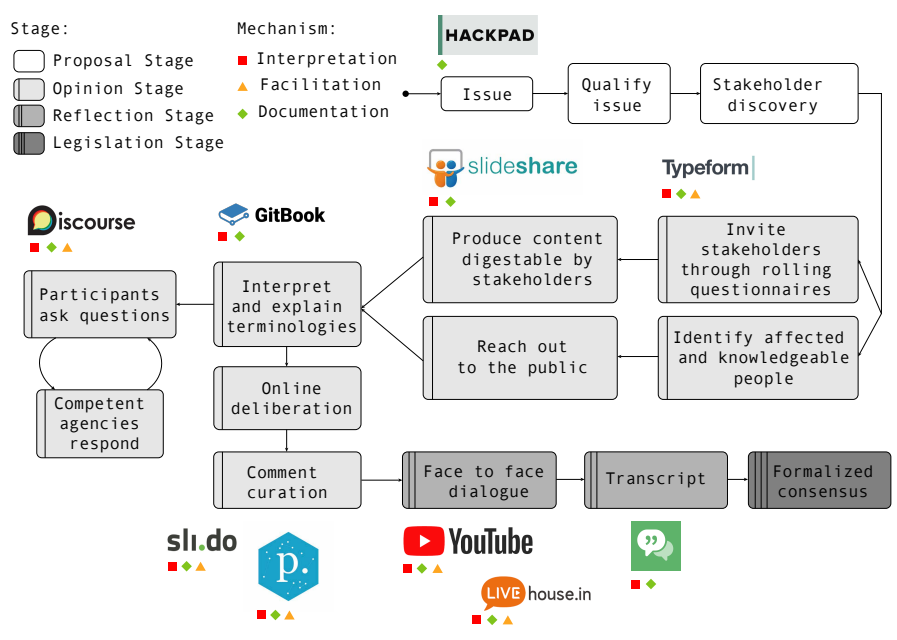

Figure 2: vTaiwan procedure and associated tools.

\section{THE UBERX CASE STUDY}

The UberX case study, one of the most acclaimed issues discussed through the vTaiwan process, illustrates and highlights the features of the vTaiwan process.

\subsection{Background}

UberX, one of the services of Uber Inc., illegally started in the mid 2014 in Taipei and gained huge popularity from the locals. Despite the fact that Uber registered as a technology company, Ministry of Transportation and Communications still deemed Uber a transport company and commanded it to obey the taxi laws. Uber refused and therefore faced a sharp increase in penalties under the Highway Act in the following years.

\subsection{Stage 1: Proposal Stage}

In 2015, under the request of several government authoritiesincluding the Ministry of Transportation and Communications, the Ministry of Economic Affairs and the Ministry of Finance-to regulate UberX's entry into Taiwan, in accordance with consensus from the vTaiwan community[2] on the topic selection, vTaiwan started the UberX case as the $12^{\text {th }}$ open consultation topic.

\subsection{Stage 2: Opinion Stage}

UberX was the first vTaiwan case that used Pol.is.[30] The Pol.is opinion survey spanned from July $15^{\text {th }}$ to August $15^{\text {th }}$. First, the community interpreted legal jargon to write the topic description and then posted default statements in plain language to initiate the discussion. Users could vote "agree", "disagree" or "pass" as a response to existing statements in Pol.is survey and/or contribute more statements. The Pol.is algorithm then identified different opinion groups based on voting patterns and the results could be accessed in a real-time report.[10]

Four broad opinion groups first emerged: taxi drivers, UberX drivers, UberX passengers and non-UberX passengers. The four groups quickly merged into two: the Group 1, representing $45 \%$ of the total participants and the Group 2, representing 55\% of the 
total participants. $87 \%$ of the participants in Group 1 identified with the following statement: "I think it is the responsibility of the Ministry to actively outlaw unlicensed passenger vehicles." $93 \%$ of the participants of Group 2 took a completely different side as they support the following statement: "Currently, the only way for traditional taxis to survive is to join a taxi fleet. This is not due to a government policy, and UberX has subverted this unwritten rule. I think it is quite awesome!" One of the majority opinions ( $95 \%$ of total participants) that were agreed upon by most participants stated: "The government should leverage this opportunity to challenge the taxi industry to improve their management \& quality control system, so that drivers \& riders would enjoy the same quality service as UberX's".

By the fourth week, participants had contributed a coherent set of reflections, expectations and suggestions, successfully forming a coherent agenda for stakeholders to respond to. In total, 925 participants took the survey; 145 comments entered into the Pol.is survey and 31,115 votes were gathered. Based on the Pol.is results, the need to regulate UberX and protect established private-public transportation was agreed upon.

\subsection{Stage 3: Reflection Stage}

In the reflection stage, online participants joined a two-hour livestreamed in-person consultation with academic and industry experts, active online users from the Pol.is survey and representatives from the following four stakeholders:

- Association of Taxi Drivers in Taipei-the main city in which UberX operated;

- Taiwan Taxi-the country's foremost taxi fleet;

- Uber Inc.-on which over USD\$1M of penalties were imposed for its illegal operations with unregistered cars;

- The Ministries of Transportation and Communications, Economic Affairs, and Finance.

The meeting combined the results from the Pol.is survey with deeper discussion and idea exchanges. 1,875 people participated on livecast. Stakeholders agreed that Uber had to comply with legal requirements of insurance and driver training. Not only Uber but also existing Taxi services agreed to expand their services in response to market demands for ride-hailing service.

\subsection{Stage 4: Legislation Stage}

On May $23^{\text {rd }}, 2016$, the administration pledged to ratify all the Pol.is consensus and to amend the Regulation on Automobile Transportation Management[11], which came into effect on October $25^{\text {th }}, 2016$ as follows:

(1) Taxis no longer need to be painted yellow.

(2) High-end app-based Taxis are free to operate as long as they do not undercut existing taxi fare.

(3) App-based dispatch systems must display car and driver identification, estimated fare, and customer rating.

(4) Per-ride taxation is required to report to the Ministry of Finance.

\subsection{Conclusion}

Governments around the globe are facing increasing challenges in regulating Uber. The UberX case study shows that the vTaiwan process offers a way to address this challenge by blending the views of the different stakeholders (citizens, taxi drivers, Uber Inc., etc.) to ensure that the essential elements of each stakeholder's view have not been ignored in crafting regulation. Due to the vTaiwan process, Uber has transformed its business model in Taiwan, and become a legal app-dispatch company. This contrasts with many other countries, where Uber has recently been banned[25].

\section{DISCUSSION}

"vTaiwan culture" is drawn from open source culture, strongly adhering to principles of transparency and collaboration, creating environments of trust, self-organization and distributed responsibility. The design used to support these principles is an adhocratic model[22]. The defining feature of this model is that it values action over formal authority. For example, when facing a difficult decision, the default in a bureaucracy is to defer to a senior colleague. The default in an adhocracy is to experiment-to try a course of action, receive feedback, make changes, and review progress[21]. Operating within this adhocratic framework, the vTaiwan process has been, and continues to be, iteratively crafted by the community, supported by open source technologies.

Despite its numerous successes in solving complex problems on political issues, vTaiwan does face many challenges. Although each citizen can submit a topic to vTaiwan, very few cases are proposed by citizens. As an ongoing institutionalized process funded by the government, vTaiwan experiment struggles to retain autonomy in the selection of topics. If a government authority refuses to discuss a public issue, the sensitive topic will not go through the vTaiwan process. Moreover, not all cases on vTaiwan lead to regulation reform. For example, the draft bill of selling liquor online was rejected by the Legislative Yuan due to its conflict with existing e-liquor policy. vTaiwan timeline and institutional timeline are desynchronized, thus public servants sometimes struggle to get used to the instability and unpredictability. For instance, a small revision of a questionnaire takes more than half a day to confirm inside the government, while it only takes several minutes to carry out at a mini hackathon.

vTaiwan struggles to balance between retaining the freedom to experiment and regulating the process of participation and collaboration. Starting January 2017, Taiwanese central government began discussions to institutionalize a rulemaking process inspired by vTaiwan.[3] Experimenting between institutionalizing or deinstitutionalizing a open consultation model is one of the future directions of vTaiwan.

vTaiwan's future work will address these struggles and continuously learn from the ongoing experiments. Despite challenges, vTaiwan is a feasible model of decentralized consultation for society. vTaiwan has potential to correct the political polarization and stands as an alternative to the bureaucratic model, serving as the voice of the relevant public, and has also fostered a collaborative culture among the public and private sectors. 


\section{REFERENCES}

[1] CeRI, Cornell E-Rulemaking Initiative. http://www.lawschool.cornell.edu/ceri/ index.cfm. Accessed: 31.01.2018.

[2] Consensus from the vTaiwan Community. https://audreyt.typeform.com/report/ vj8HJw/rECm. Accessed: 18.01.2018.

[3] Digital Communication Bill. https://www.ncc.gov.tw/chinese/files/17012/3864_ 36859_170124_1.pdf. Accessed: 31.01.2018.

[4] Discourse, home page. Accessed: 20.12.2017.

[5] g0v, home page. http://g0v.tw/en-US. Accessed: 31.12 .2017

[6] Hackpad, home page. https://hackpad.tw/. Accessed: 21.01.2017.

[7] Join, home page. https://join.gov.tw. Accessed: 30.12.2017.

[8] LIVEhouse.in, home page. https://livehouse.in/. Accessed: 20.12.2017.

[9] Pol.is, GitHub. https://github.com/pol-is. Accessed: 20.12.2017.

[10] Pol.is Report of Uber. https://pol.is/report/r32beaksmhwesyum6kaur. Accessed: 31.01.2018. The numbers in the report are marginally higher than those reported in this paper due to the polis survey being open to the public even after the opinion stage.

[11] Regulation on Automobile Transportation Management. http://law.moj.gov.tw/ LawClass/LawContent.aspx?PCODE=K0040003. Laws \& Regulations Database of the Republic of China, Accessed: 01.02.2018.

[12] SlideShare, home page. https://www.slideshare.net/. Accessed: 01.02.2018.

[13] Sli.do, home page. https://www.sli.do/. Accessed: 20.12.2017.

[14] "Talk to Taiwan-Jaclyn Tsai" Internet World Impacting on Traditional Laws: How Many Steps Should Taiwan Take to Catch up? https://www.youtube.com/ watch? $\mathrm{v}=\operatorname{dig} 0 \mathrm{WjnD} 3 \mathrm{pQ} \& \mathrm{t}=10 \mathrm{~m}$. Accessed: 04.01.2018.

[15] Typeform, home page. https://www.typeform.com/. Accessed: 20.12.2017.

[16] vTaiwan, home page. https://vtaiwan.tw. Accessed: 30.12.2017.

[17] vTaiwan Project. https://www.overleaf.com/read/kvmdcwxzqkfc. This paper was collaboratively written by vTaiwan contributors. Our thanks to Liz Barry, Cheng-En Hsieh, and all vTaiwan contributors. Accessed: 04.07.2018.

[18] Abdi, H., and Williams, L. J. Principal Component Analysis. Wiley Interdisciplinary Reviews: Computational Statistics 2(4) (2010), 433-459.

[19] Agar, N. Don't Worry about Superintelligence. Fournal of Evolution and Technology 26(1) (2016), 73-82

[20] Barberá, P., Jost, J. T., Nagler, J., Tucker, J. A., and Bonneau, R. Tweeting from Left to Right: Is Online Political Communication more than an Echo Chamber? Psychological Science 26(10) (2015), 1531-1542.

[21] Birkinshaw, J. Ridderstråle, J. Adhocracy for an Agile age. Organization Science 22(5) (2010), 1286-1296.

[22] Dolan, E. T. Revisiting Adhocracy: From Rhetorical Revisionism to Smart Mobs. fournal of Futures Studies 15(2) (2010), 33-50.

[23] Fell, D. Taiwan's Social Movements under Ma Ying-jeou: From the Wild Strawberries to the Sunflowers. Routledge, Oxford, 2017.

[24] Goertzel, B., And Pitt, J. Nine Ways to Bias Open-Source Artificial General Intelligence toward Friendliness. Fournal of Evolution and Technology 22(1) (2012) $116-131$

[25] Hao, K. Map: All the Places Where Uber is Partially or Fully Banned. https://qz. com/1084981/map-all-the-places-where-uber-is-partially-or-fully-banned. Accessed: 18.01.2018.

[26] Hotelling, H. Analysis of a Complex of Statistical Variables into Principal Components. Fournal of Educational Psychology 24(6) (1933), 417-441.

[27] Kelty, C. Geeks, Social Imaginaries, and Recursive Publics. Cultural Anthropology 20(2) (2005), 185-214

[28] Lawrence, E., Sides, J., And Farrell, H. Self-Segregation or Deliberation? Blog Readership, Participation, and Polarization in American Politics. Perspective on Politics 8(1) (2010), 141-157.

[29] MacQueEn, J. Some Methods for Classification and Analysis of Multivariate Observations. Proceedings of the Fifth Berkeley Symposium on Mathematical Statistics and Probability 1 (1967), 281-297.

[30] Megill, C. Pol.is in Taiwan. Medium https://blog.pol.is/ pol-is-in-taiwan-da7570d372b5. Accessed: 19.12.2017.

[31] Rietbergen-McCracken, J. Participatory Policy Making. CIVICUS http://www. civicus.org/documents/toolkits/PGX_F_ParticipatoryPolicy\%20Making.pdf. Ac cessed: 19.12.2017.

[32] Rosanvallon, P. Counter-Democracy: Politics in an Age of Distrust. Cambridge University Press, Cambridge, 2008.

[33] Solivan, J., And Farina, R. C. Regulation Room: How the Internet Improves Public Participation in Rulemaking. Cornell e-Rulemaking Initiative Publications (2013), 57-62.

[34] Stanfield, R. B. The Art of Focused Conversation: 100 Ways to Access Group Wisdom in the Workplace. New Society Publishers, Canada, 2000.

[35] Tseng, P. Y., AND LeE, M. C. Taiwan Open Government Report. Open Culture Foundation, Taipei, 2017.

[36] Vicario, M. D., Vivaldo, G., Bessi, A., Zollo, F., Scala, A., Caldarelli, G., AND Quatrociocchi, W. Echo Chambers: Emotional Contagion and Group Polarization on Facebook. Scientific Reports 6 (2016).
[37] YEH, J. R. Towards E-Government: The Challenge of Disclosure of Information and Administrative Procedures. Taiwan Law Society Review 19 (1998), 1-48. 